\title{
Maxwellian stresses and electromagnetic forces that arise from them in vacuum and media
}

\author{
I. Campos \\ Departamento de Física, Facultad de Ciencias, Universidad Nacional Autónoma de México, \\ México, 04000, Ciudad de México. \\ e-mail: iecampos@prodigy.net.mx \\ J. L. Jiménez \\ Departamento de Física, División de Ciencias Básicas e Ingeniería, Universidad Autónoma Metropolitana, Iztapalapa, \\ Av. San Rafael Atlixco \# 186, Col. Vicentina, Apartado Postal 21-463, México, 04000, Ciudad de México. \\ e-mail: jlj@xanum.uam.mx \\ J. A. E. Roa-Neri \\ Área de Física Teórica y Materia Condensada, División de Ciencias Básicas e Ingeniería, \\ Universidad Autónoma Metropolitana Unidad Azcapotzalco, \\ Apartado Postal 16-225, México, 02011 Ciudad de México.e-mail: rnjae@correo.azc.uam.mx
}

Received 19 May 2020; accepted 16 June 2020

Faraday and Maxwell conceived the electrostatic and magnetostatic fields as lines of force filling the space around charges and currents, with or without matter. They also established that there are tensions along the lines of force, and compressions around these lines. These ideas are formalized with a stress tensor, whose divergence is a force density. We follow these ideas to show the origin of the electric forces that arise in the case of a dielectric slab partially introduced into a parallel-plate capacitor, and the origin of the magnetic forces that arise in the case of a magnetizable bar partially introduced into a solenoid. We find that these forces have their origin in the abrupt change in permittivity and permeability which produce a difference of pressures at interfaces. This approach permits us to analyze the similarities and differences between both cases. Advanced undergraduate and graduate students can get interesting insights into the electromagnetic forces exerted by electrostatic and magnetostatic fields in vacuum and matter.

Keywords: Maxwell equations; electromagnetic balance equations; Maxwell stresses; electromagnetic force densities.

Faraday y Maxwell concibieron los campos electrostáticos y magnetostáticos como líneas de fuerza llenando el espacio alrededor de cargas y corriente, con o sin materia. Ellos establecieron que hay tensiones a lo largo de las líneas de fuerza, y compresiones alrededor de estas líneas. Estas ideas se formalizan con el tensor de esfuerzos, cuya divergencia es una densidad de fuerza. Aquí se siguen estas ideas para mostrar el origen de las fuerzas eléctricas que surgen cuando una barra de dieléctrico se introduce parcialmente en un condensador de placas paralelas, así como para exponer el origen de las fuerzas magnéticas que surgen en el caso de una barra magnetizable parcialmente introducida en un solenoide. Se encontró que estas fuerzas tienen su origen en un cambio abrupto en la permitividad y permeabilidad que produce una diferencia de presiones en la interface. Este enfoque permite analizar las similitudes y diferencias entre ambos casos. Los estudiantes no graduados y graduados pueden adquirir una percepción interesante sobre las fuerzas electromagnéticas ejercidas por campos electrostáticos y magnetostáticos en el vacío y en la materia.

Descriptores: Ecuaciones de Maxwell; ecuaciones de balance electromagnético; esfuerzos de Maxwell; densidades de fuerza electromagnéticas.

PACS: 03.50De; $41.20 \mathrm{Jb}$

DOI: https://doi.org/10.31349/RevMexFisE.18.3

\section{Introduction}

In elementary and intermediate textbooks on electromagnetism it is usually affirmed that, in electrostatics, the introduction of the concept of field is somewhat artificial: "When dealing with static fields, the field concept is, in fact, superfluous" [1]. The basic law of interaction between charged bodies, Coulomb's law, is a kind of action at a distance, and the force between point charges acts in the direction of the line joining these. The field is introduced, in electrostatics, probing it with a test charge $q$, and defining it as $\boldsymbol{E}=\boldsymbol{F} / q$. It is also said that the notion of a field is necessary only in treating time-varying fields. Thus, the only force considered in electrostatics is the electric part of Lorentz's force law, $\boldsymbol{F}=q \boldsymbol{E}$, therefore there is only a force tangent to the lines of force. From this, it follows that a uniform electrostatic field can only rotate a dipole and cannot exert a net force on it. In a vacuum, only a non-uniform electrostatic field can exert a net force on a dipole. This idea is extrapolated to the electrostatic force on materials, and it is assumed that only a non-uniform field can exert a net force on the polarizable matter. This is the conception usually adduced to explain the force on a dielectric slab partially introduced into a parallel-plate capacitor: it arises from the action of the non-uniform fringing field out- 
side the capacitor on the electric dipoles of the medium [2-6]. This explanation is, however, incompatible with the accepted calculation, which is done with the uniform field inside the capacitor. Margulies [2] even asks how is it possible that a uniform field can exert a force orthogonal to it. In contrast, in the case of a magnetizable bar partially introduced into a solenoid, there is no objection to the fact that a uniform magnetic field can only rotate a magnetic dipole, without exerting a net force on it. Here it seems normal that a uniform field can exert a net force on the magnetic dipoles of a magnetizable material.

Faraday and Maxwell, however, conceived the electrostatic and magnetostatic fields as force lines filling the space around charged bodies and currents, with tensions along the lines of force and pressures around these lines, that is force is transmitted through a surface by stress tensor. Thus, we have the possibility of electrostatic forces orthogonal to the lines of force, and not only forces tangential to these lines. These ideas are exposed in well-known textbooks [7-9].

In the present paper, we analyze the forces that arise in the interaction of electrostatic and magnetostatic fields with the polarizable and magnetizable matter. We show that the formalism of an electromagnetic momentum balance equation with a particular stress tensor provides interesting insights into the origin of the forces that arise in the interaction field-matter. We take as an example the interaction that arises in the elementary problem of the force that pulls a dielectric slab into a charged parallel-plate capacitor and compares it with the pulling of a magnetizable bar into a solenoid. These effects are usually considered analogous. However, our analysis shows that these electrostatic and magnetostatic effects are not analogous. In the electrostatic case, the force arises from the pressures orthogonal to the lines of force, while in the magnetostatic case, the force arises from the tension along the magnetostatic lines of force. Indeed, we have proposed elsewhere [10] a magnetostatic device that we argue is really analogous to the case of a dielectric slab inside a capacitor. In this device, the force arises from the magnetostatics pressures, as is the case with the dielectric slab inserted into a capacitor.

Our analysis is based on the original ideas of Faraday and Maxwell, elaborated formally in classical texts as those by Becker [7], Stratton [8] and Panofsky, and Phillips [9]. We consider that the mentioned effects are better explained with the introduction of an electromagnetic momentum balance equation, which relates the divergence of a stress tensor with a force density. Indeed, we have that Noether's theorem establishes the equivalence of the equations of motion of the fields and conservation laws or momentum balance equations $[11,12]$. We have derived several momentum balance equations transforming the macroscopic Maxwell equations into balanced equations using vector and dyad identities [13-14]. This approach permits a unified treatment of vacuum and media.

The results and interpretations obtained differ from the usual explanation of the origin of these forces, exposed in many textbooks and articles. Our method, however, is wellfounded and provides insights that, we think, deserves consideration.

\section{Force density in a vacuum as a divergence of a stress tensor}

According to the original ideas of Faraday and Maxwell [15], the notion of field, even in static conditions, is more conveniently illustrated with a stress tensor, whose divergence is a force density. They are related through a balance equation of the form

$$
\boldsymbol{f}_{e}=\nabla \cdot \overleftrightarrow{\boldsymbol{T}}_{\text {evacuum }}
$$

For electrostatics the tensor that satisfies this balanced equation is

$$
\begin{aligned}
\overleftrightarrow{\boldsymbol{T}}_{e \text { vacuum }} & =\epsilon_{0}\left(\boldsymbol{E} \boldsymbol{E}-\frac{1}{2} E^{2 \overleftrightarrow{\boldsymbol{I}}}\right) \\
& =\left(\boldsymbol{D}_{0} \boldsymbol{E}-\frac{1}{2}\left(\boldsymbol{D}_{0} \cdot \boldsymbol{E}\right) \overleftrightarrow{\boldsymbol{I}}\right)
\end{aligned}
$$

Usually $[9,16] \mathrm{Eq}$. (1) is derived starting with Lorentz's force density for electrostatics,

$$
\boldsymbol{f}_{e}=\rho \boldsymbol{E},
$$

in which the charge density is substituted from $\nabla \cdot \epsilon_{0} \boldsymbol{E}=\rho$, and with vector identities the tensor Eq. (2) is obtained.

The balance Eq. (1) allows to calculate the force either as a volume integration of the force density or, using Gauss's theorem, as a surface integral of the stress tensor. This last method indicates that the resulting force is not necessarily in the direction of the field. We have then that the force is

$$
\boldsymbol{F}=\int \boldsymbol{f} d V=\oint \overleftrightarrow{\boldsymbol{T}} \cdot d \boldsymbol{S}
$$

In some advanced texts of electromagnetism [7-9], the symmetric stress tensor is reduced to three components by transformation to principal axes, resulting in tension along the lines of force of magnitude $(1 / 2) \epsilon_{0} \boldsymbol{E}^{2}$, and compression of magnitude $-(1 / 2) \epsilon_{0} \boldsymbol{E}^{2}$ along two orthogonal axes around the lines of force.

A simple example of the calculation of a force using this approach is the derivation of Coulomb's law in a vacuum. This law is obtained [7,9] integrating the stress tensor over an infinite surface orthogonal to the line joining the equal point charges, at the middle point, closed at infinity, where the field is zero. It is important to note that the field used to build the stress tensor is the field of both charges, that is, the total field. This may seem strange since, in Lorentz's force density, the field is only the external field. Let us remember that the electromagnetic field in any region is determined partially by charges inside the region (inhomogeneous solutions), and partially by charges outside the region (homogeneous solutions). Therefore, when in the usual derivation of Eq. (1) the 
charge density appearing in Gauss's law, $\epsilon_{0} \boldsymbol{E}=\rho$, is substituted in the Lorentz force density, $\boldsymbol{f}=\rho \boldsymbol{E}_{\text {ext }}$, the stress tensor results with a mixture of external and total fields. Therefore, the usual deduction [16] is inconsistent. However, Eq. (1) can be deduced from Maxwell's equations in a vacuum without using Lorentz's force density as a starting point [17]. In this way, the inconsistency is avoided because the total field is always used. It is noteworthy that in these static conditions either with the inconsistent deduction of the balance equation, or with the balance equation with total fields, in the resulting electrostatic force density appears only the external field.

This is because the balance equation gives the force on any region that encloses one of the charges. The region used above to derive Coulomb's law, the semi-space, is just convenient for the calculation because of the symmetry. However, a small spherical surface surrounding one of the charges must also give the force density, though the lack of symmetry impedes a calculation. In this small sphere, the self-field is symmetrical and does not contribute to the force density, remaining only the external field. The same effect occurs when, in a uniform electrostatic field, a point charge is introduced. The stress tensor contains the total field, but in the net force density, only the external field appears [18].

The formalism of a balance equation implies that the total electromagnetic force on a given region is the force transmitted through the surface that encloses the given region. The force can also be calculated with a volume integration of the force density over the volume of the region, involving the total fields. The result obtained before for Coulomb's law leads then to two different interpretations, either the force is transmitted through stresses of the total field, or the force arises from the action of an external field on charges. In the first case, the infinite force that would arise from the interaction of the self-field with a finite point charge does not arise, since it is the total field that determines the force.

In the case of equal charges, the lines of force are parallel to this surface, so that the force is orthogonal to the lines of force, while for charges of different sign the force arises from the tension, which is tangent to the lines of force and therefore the force is orthogonal to this surface. This view, therefore, implies that the force may be exerted not only in a direction tangent to the lines of force, as usually assumed in most texts, but also in a direction orthogonal to the lines of force.

We have then a general method to calculate the force on charges, which consists of integrating the stress tensor over a closed surface surrounding the volume of interest.

\section{Electrostatic forces on material media}

The calculation of forces on media can also be carried out by using a stress tensor. We have shown elsewhere [19] that a particular form of the macroscopic Maxwell equations can be transformed through vector and dyadic identities into a momentum balance equation whose electrostatic part is

$$
\begin{aligned}
\nabla \cdot & \left\{\boldsymbol{D} \boldsymbol{E}-\frac{1}{2} \boldsymbol{I}(\boldsymbol{D} \cdot \boldsymbol{E})\right\}=\rho \boldsymbol{E} \\
& +\frac{1}{2}[(\nabla \boldsymbol{E}) \cdot \boldsymbol{D}-(\nabla \boldsymbol{D}) \cdot \boldsymbol{E}]=\boldsymbol{f}_{\text {Max elec media }} .
\end{aligned}
$$

It is important to emphasize that being derived from the macroscopic Maxwell equations, the fields appearing in this equation are the total fields, avoiding the inconsistency discussed before. In this equation, we can identify the stress tensor

$$
\overleftrightarrow{\boldsymbol{T}}_{e \mathrm{mat} \text { media }}=\boldsymbol{D E}-\frac{1}{2} \boldsymbol{I}(\boldsymbol{D} \cdot \boldsymbol{E}) .
$$

Then we have, as in a vacuum,

$$
\overleftrightarrow{\boldsymbol{F}}_{\text {emat media }}=\oint_{s=\partial V} d \boldsymbol{S} \cdot \overleftrightarrow{\boldsymbol{T}}_{\text {e mat media }}
$$

Thus, the balance equation permits us to calculate the force either as a surface integration of the stress tensor or as a volume integration of the force density.

Since we are interested in the electrostatic forces acting on polarizable material media, we set $\rho E=0$. There is yet a force acting on matter. If we have a linear medium, the constitutive relation is

$$
\boldsymbol{D}=\epsilon_{0} \epsilon_{r} \boldsymbol{E} .
$$

Then the tensor Eq. (6) is

$$
\overleftrightarrow{\boldsymbol{T}}_{e \text { mat media }}=\epsilon_{0} \epsilon_{r}\left(\boldsymbol{E} \boldsymbol{E}-\frac{1}{2}\left(E^{2}\right) \boldsymbol{I}\right)
$$

Thus, if we consider a small element of area $\sigma$ represented by a unit vector $\hat{\boldsymbol{n}}$ orthogonal to it, on this small area the field can be considered constant, and then

$$
\boldsymbol{F}=\int_{\sigma} \overleftrightarrow{\boldsymbol{T}}_{e \text { mat media }} \cdot \hat{\boldsymbol{n}} d A=\overleftrightarrow{\boldsymbol{T}}_{e \text { mat media }} \cdot \hat{\boldsymbol{n}} \sigma
$$

is the force transmitted across this element of the area.

If the surface is orthogonal to $\boldsymbol{E}, \hat{\boldsymbol{n}}$ is parallel to $\boldsymbol{E}$ and

$$
\overleftrightarrow{\boldsymbol{T}}_{e \text { mat media }} \cdot \hat{\boldsymbol{n}} \sigma=\epsilon_{0} \epsilon_{r} \frac{1}{2}\left(E^{2}\right) \hat{\boldsymbol{n}} \sigma,
$$

giving a force parallel to $\boldsymbol{E}$.

If the surface is parallel to $\boldsymbol{E}$, then $\hat{\boldsymbol{n}}$ is orthogonal to $\boldsymbol{E}$ and

$$
\overleftrightarrow{\boldsymbol{T}}_{e \text { mat media }} \cdot \hat{\boldsymbol{n}} \sigma=-\epsilon_{0} \epsilon_{r} \frac{1}{2}\left(E^{2}\right) \hat{\boldsymbol{n}} \sigma,
$$

giving now a force orthogonal to $\boldsymbol{E}$. Then the force exerted on charges, currents, or matter inside a closed surface is given by the surface integral of the stress tensor, with the normal $\hat{n}$ pointing outwards.

These ideas can be applied to the calculation of the force exerted on a dielectric slab partially introduced into a charged 
parallel-plate capacitor. Our approach explains how a uniform electrostatic field can exert a force orthogonal to it, eliminating in this way the usual inconsistency in arguing that it is the non-uniform fringing field the origin of the force, while the field used in the calculation is the uniform field inside the capacitor. We can see that Maxwell's theory allows forces orthogonal to the lines of force, though it may seem strange, since we are usually familiar only with forces tangent to the lines of force, due to the tension along these lines. Our treatment of the force on a dielectric slab will make clear this point.

Let us assume that the uniform field inside the capacitor is $\hat{\mathbf{i}}$, while the slab moves in the $\hat{\boldsymbol{k}}$ direction.

Then the constitutive relation is Eq. (8), and the stress tensor results

$$
\overleftrightarrow{\boldsymbol{T}}=\varepsilon_{0} \varepsilon_{r} E^{2}\left[\hat{\mathbf{i}} \mathbf{i}-\frac{1}{2} \overleftrightarrow{\boldsymbol{I}}\right]
$$

where

$$
\overleftrightarrow{\boldsymbol{I}}=\hat{\mathbf{\imath}} \mathbf{i}+\hat{\mathbf{\jmath}} \mathbf{\jmath}+\hat{\boldsymbol{k}} \hat{\boldsymbol{k}}
$$

Now we choose a closed surface around the interface, forming a parallelepiped with planes close and parallel to the interface. These surfaces are represented by unit vectors $\hat{\boldsymbol{k}}$ and $-\hat{\boldsymbol{k}}$.

The closed surface is completed with a "ribbon" joining the parallel planes. Given this simple geometry, only the integral of the stress on the planes $\hat{\boldsymbol{k}}$ and $-\hat{\boldsymbol{k}}$ contribute. Then, substituting Eq. (13) in Eq. (9) results in

$$
\begin{aligned}
\boldsymbol{F} & =\int_{\sigma \text { medium }}(-\hat{\boldsymbol{k}}) d S \cdot \epsilon_{0} \epsilon_{r} E^{2}\left[\hat{\mathbf{i}}-\frac{1}{2} \overleftrightarrow{\boldsymbol{I}}\right] \\
& -\int_{\sigma \text { medium }} \hat{\boldsymbol{k}} d S \cdot \epsilon_{0} E^{2}\left[\hat{\mathbf{i}}-\frac{1}{2} \overleftrightarrow{\boldsymbol{I}}\right]
\end{aligned}
$$

and therefore, the force is

$$
\boldsymbol{F}=\frac{1}{2} \epsilon_{0} E^{2}\left(\epsilon_{r}-1\right) \int \hat{\boldsymbol{k}} d S .
$$

Since we have that

$$
\int \hat{\boldsymbol{k}} d S=\hat{\boldsymbol{k}} L h,
$$

where $L h$ is the cross area of the slab, and expressing the relative permittivity in terms of the susceptibility, we finally obtain

$$
\boldsymbol{F}=\frac{1}{2} \epsilon_{0} \chi_{e} L h E \hat{\boldsymbol{k}},
$$

which is the accepted result. Our treatment shows clearly that the force is exerted at the interface, and the fringing field is irrelevant.

In this case, the electrostatic field is considered uniform inside the capacitor, facilitating the calculation. This approach contradicts the usual explanation that the force arises from the action of the fringing field outside the capacitor. It is noteworthy that, in the usual approach, the calculation is done with the uniform field, and some authors [20,21] explicitly neglect the fringing field.

\section{The force density}

The force Eq. (18) that is exerted on a dielectric slab inside a parallel plate capacitor can also be obtained by a volume integration of a force density. Usually, this force density is obtained as a gradient of an energy density. The change in energy due to the introduction of the dielectric slab is

$$
W=-\int_{V} d V \boldsymbol{P} \cdot \boldsymbol{E}=-\int_{V} d V u .
$$

The energy density u implies a force density (at a constant charge)

$$
f=-\nabla(\boldsymbol{P} \cdot \boldsymbol{E}) .
$$

This force density leads to the accepted result Eq. (18), and is taken as equivalent to a force density $(\boldsymbol{P} \cdot \boldsymbol{\nabla}) E$, which applies in the case of a non-uniform field. However, if we consider the identity

$$
\begin{aligned}
\boldsymbol{\nabla}(\boldsymbol{a} \cdot \boldsymbol{b}) & =(\boldsymbol{a} \cdot \boldsymbol{\nabla}) \boldsymbol{b}+(\boldsymbol{b} \cdot \boldsymbol{\nabla}) \boldsymbol{a} \\
& +\boldsymbol{a} \times(\boldsymbol{\nabla} \times \boldsymbol{b})+\boldsymbol{b} \times(\boldsymbol{\nabla} \times \boldsymbol{a}),
\end{aligned}
$$

taking $\boldsymbol{a}=\boldsymbol{P}$ and $\boldsymbol{b}=\boldsymbol{E}$, we get

$$
\begin{aligned}
\boldsymbol{f} & =-\frac{1}{2}\{(\boldsymbol{p} \cdot \boldsymbol{\nabla}) \boldsymbol{E}+(\boldsymbol{E} \cdot \boldsymbol{\nabla}) \boldsymbol{P} \\
& +\boldsymbol{p} \times(\boldsymbol{\nabla} \times \boldsymbol{E})+\boldsymbol{E} \times(\boldsymbol{\nabla} \times \boldsymbol{P})\} .
\end{aligned}
$$

Since we have a uniform electrostatic field inside the capacitor, the following conditions apply:

$$
\begin{aligned}
(\boldsymbol{P} \cdot \boldsymbol{\nabla}) \boldsymbol{E} & =0, \\
\boldsymbol{\nabla} \times \boldsymbol{E} & =0 .
\end{aligned}
$$

Now, for linear media, the polarization is proportional to the electrostatic field and is discontinuous at the interface. Then,

$$
(\boldsymbol{E} \cdot \boldsymbol{\nabla}) \boldsymbol{P}=\left(E_{x} \partial_{x}\right) \hat{\mathbf{1}} P(Z)=0,
$$

and Eq. (22) is in this way reduced to

$$
\boldsymbol{f}=-\frac{1}{2}(\boldsymbol{E} \times(\boldsymbol{\nabla} \times \boldsymbol{P})) .
$$

This force density may seem unfamiliar, but we have shown above, and in [22] that it is equivalent to Eq. (20), from which it is derived using vector identities and the conditions of the problem. We have also shown elsewhere [22] that this force density leads to the known expression for the force, Eq. (18). With this approach, it can be seen that the force on dielectrics is not only $(\boldsymbol{P} \cdot \boldsymbol{\nabla}) \boldsymbol{E}$, which holds when the field is non-uniform and therefore is zero in the present case. We have also the force density Eq. (26), which applies even in the case of a uniform field. Therefore, the non-uniform fringing field is irrelevant, as some authors explicitly say [20,21]. This is an implication of Maxwell's theory. 


\section{Magnetostatic forces in the vacuum}

The force and force density associated with magnetostatic fields and its relation to a stress tensor present analogies and differences to the electrostatic case. In a vacuum, the magnetic force density is given by

$$
\boldsymbol{f}_{m}=\boldsymbol{J} \times \boldsymbol{B},
$$

where $\boldsymbol{B}$ is not produced by $\boldsymbol{J}$ so that it is considered external. Of course, the force is exerted on $\boldsymbol{J}$.

The usual way of deriving a stress tensor [16] is substituting the current density J from Ampére's law (where the total magnetic field must appear) and using some vector identities, one gets

$$
\boldsymbol{f}_{m}=\nabla \cdot \overleftrightarrow{\boldsymbol{T}}_{m \text { vacuum }}
$$

where

$$
\begin{aligned}
\overleftrightarrow{\boldsymbol{T}}_{m \text { vacuum }} & =\frac{1}{\mu_{0}}\left(\boldsymbol{B} \boldsymbol{B}-\frac{1}{2} B^{2} \overleftrightarrow{\boldsymbol{I}}\right) \\
& =\left(\boldsymbol{B} \boldsymbol{H}_{0}-\frac{1}{2}\left(\boldsymbol{B} \cdot \boldsymbol{H}_{0}\right) \overleftrightarrow{\boldsymbol{I}}\right)
\end{aligned}
$$

since in vacuum

$$
\boldsymbol{B}=\mu_{0} \boldsymbol{H}_{0}
$$

The volume integration of Eq. (28) results

$$
F_{m}=\oint_{S=\partial V} d \boldsymbol{S} \cdot \overleftrightarrow{\boldsymbol{T}}_{m \text { vacuum }}
$$

where Gauss's theorem was used.

Again, as it happens in the electrostatic case, it must be noted that when Eq. (27) and Eq. (29) are inserted in Eq. (28) the magnetic field on the left side must be external, but on the right side must be the total one.

This inconsistency can be avoided with a balanced equation, obtained transforming Maxwell's equations with total fields.

Again, the decomposition of the stress tensor into principal axes results, in a vacuum, in a tension $(1 / 2) \mu_{0} H^{2}$ along the lines of force, and a compression $-(1 / 2) \mu_{0} H^{2}$ in two orthogonal axes around the lines of force. The compression may produce forces orthogonal to the lines of force, while the tension produces forces along the lines of force.

\section{Magnetostatic forces in material media}

The magnetostatic forces that arise from the interaction of magnetostatic fields and magnetizable matter present some analogies with the electrostatic case, but there are also some differences. In this case, the correspondence $\boldsymbol{E} \rightarrow \boldsymbol{B}$ and $\boldsymbol{D} \rightarrow \boldsymbol{H}$ usually suggested to obtain the analogy to the electrostatic case $[20,16]$ does not work. In the case, of the magnetic field, we are acquainted with the fact that the force on currents is orthogonal to the field and current, that a uniform magnetic field can only align magnetic dipoles. It cannot exert a net force on them. However, in the case of a magnetizable bar partially introduced into a solenoid, this is what happens, and it is accepted as a fact, but it lacks an explanation. We propose an explanation by means of a stress tensor, which appears in an electromagnetic momentum balance equation derived from the macroscopic Maxwell equations. As in the electrostatic case, this tensor can be decomposed in tensions along the lines of force, and compressions around these lines.

This balance equation is obtained elsewhere $[17,19]$ and is analogous to the electrostatic case,

$$
\begin{aligned}
\boldsymbol{\nabla} & \cdot\left\{\boldsymbol{B} \boldsymbol{H}-\frac{1}{2} \boldsymbol{I}(\boldsymbol{B} \cdot \boldsymbol{H})\right\}=\boldsymbol{j} \times \boldsymbol{B}+\frac{1}{2}[(\boldsymbol{\nabla} \boldsymbol{H}) \cdot \boldsymbol{B} \\
& -(\boldsymbol{\nabla} \boldsymbol{B}) \cdot \boldsymbol{H}]=\boldsymbol{f}_{\text {Max mag media }}
\end{aligned}
$$

where, for linear media with relative permeability $\mu_{r}$ we have the constitutive relation

$$
\boldsymbol{B}=\mu_{0} \mu_{r} \boldsymbol{H} .
$$

As in the electrostatic case, we have that the force can be calculated as a surface integral of the stress tensor,

$$
\boldsymbol{F}_{m \text { mat media }}=\oint_{S=\partial V} d \boldsymbol{S} \cdot \overleftrightarrow{\boldsymbol{T}}_{m \text { mat media }}
$$

where

$$
\overleftrightarrow{\boldsymbol{T}}_{m \text { mat media }}=\boldsymbol{B H}-\frac{1}{2} \boldsymbol{I}(\boldsymbol{B} \cdot \boldsymbol{H}),
$$

or as a volume integration of the force density,

$$
\begin{aligned}
\boldsymbol{F}_{m \text { mat media }} & =\int_{V} d V\{\boldsymbol{j} \times \boldsymbol{B} \\
& \left.+\frac{1}{2}[(\boldsymbol{\nabla} \boldsymbol{H}) \cdot \boldsymbol{B}-(\boldsymbol{\nabla} \boldsymbol{B}) \cdot \boldsymbol{H}]\right\} .
\end{aligned}
$$

In the present case, there are not free currents, so $\boldsymbol{J} \times \boldsymbol{B}=$ 0 , but the force on material media is not zero.

Since we are interested in the magnetostatic forces acting on magnetizable material media, we set $\boldsymbol{J}=0$. There is yet a force acting on matter. If we have a linear medium, the constitutive relation is

$$
B=\mu_{0} \mu_{r} \boldsymbol{H} .
$$

Then the tensor Eq. (35) is

$$
\overleftrightarrow{\boldsymbol{T}}_{m \text { mat media }}=\mu_{0} \mu_{r}\left(\boldsymbol{H} \boldsymbol{H}-\frac{1}{2}\left(H^{2}\right) \boldsymbol{I}\right)
$$

If we consider an element of area $A_{0}$ represented by a unit vector $\hat{\boldsymbol{n}}$ orthogonal to it, then

$$
\boldsymbol{F}=\overleftrightarrow{\boldsymbol{T}}_{m \text { mat media }} \cdot A_{0} \hat{\boldsymbol{n}}
$$


is the force transmitted across this element of the area.

If the surface is orthogonal to $\boldsymbol{H}, \hat{\boldsymbol{n}}$ is parallel to $\boldsymbol{H}$ and

$$
\overleftrightarrow{\boldsymbol{T}}_{m \text { mat media }} \cdot A_{0} \hat{\boldsymbol{n}}=-\mu_{0} \mu_{r} \frac{1}{2}\left(H^{2}\right) \hat{\boldsymbol{n}} A_{0}
$$

giving a force parallel to $\boldsymbol{H}$.

If the surface is parallel to $\boldsymbol{H}$, then $\hat{\boldsymbol{n}}$ is orthogonal to $\boldsymbol{H}$ and

$$
\overleftrightarrow{\boldsymbol{T}}_{m \text { mat media }} \cdot A_{0} \hat{\boldsymbol{n}}=-\mu_{0} \mu_{r} \frac{1}{2}\left(H^{2}\right) \hat{\boldsymbol{n}} A_{0}
$$

giving now a force orthogonal to $\boldsymbol{H}$.

These ideas can be applied to the calculation of the force exerted on a magnetizable rod partially introduced into a solenoid.

What we need now is the equations; for a solenoid with constant current $I$, equivalent to the electrostatic case, Eq. (18) derived from Eq. (34). When the solenoid is along the $z$ direction, the field is also in the $z$-direction, in the region where there is magnetizable material $(z<0)$ as well as in vacuum $(z>0)$. If we choose a closed surface around the interface, forming a thin pill-box around the interface, the surfaces have directions given by unit vectors $\hat{\boldsymbol{k}}$ and $-\hat{\boldsymbol{k}}$ and a "ribbon" joining the planes. Then the force analogous to force Eq. (18) results,

$$
F=\frac{1}{2} \mu_{0} \chi_{m} H^{2} A_{0} \hat{\boldsymbol{k}} .
$$

As mentioned before, here appears a force parallel to a uniform magnetic field that has an explanation in terms of the tension part of the stress tensor.

In comparing Eqs. (42) with the electrostatic case, Eq. (18), we can see that the usual correspondence suggested following the analogy with the electrostatic case, $\boldsymbol{E} \rightarrow \boldsymbol{B}$ and $\boldsymbol{D} \rightarrow \boldsymbol{H}$, does not lead to the correct result. This correspondence also leads to the energy with the wrong sign $[16,20]$

$$
W_{\text {Mag }}=-\frac{1}{2} \mu_{0} \int_{V} d V M \cdot H_{0} \quad \text { iwrong! } .
$$

In comparing this expression with the equivalent electric expression, some authors even call attention to the change in sign [16,20], but insist in using this correspondence [24] establish clearly, using thermodynamic arguments, that the correct expression for the energy is

$$
W_{\text {Mag }}=-\frac{1}{2} \mu_{0} \int_{V} d V M \cdot H_{0} \text { icorrect! }
$$

Therefore, we conclude that in this case the correspondence $\boldsymbol{E} \rightarrow \boldsymbol{H}$ and $\boldsymbol{D} \rightarrow \boldsymbol{B}$ is what leads to the accepted result.

That the analogy between the dielectric slab inside a capacitor and a magnetizable bar inside a solenoid is not exact can also be noted by the fact that the magnetic force density analogous to the force density Eq. (26),

$$
\boldsymbol{f}_{\text {new mag }}=-\frac{1}{2} \mu_{0} \boldsymbol{H} \times(\boldsymbol{\nabla} \times \boldsymbol{M}),
$$

does not lead to the accepted result. We can show this point starting with the force density that leads to the accepted force,

$$
\boldsymbol{f}_{m}=-\boldsymbol{\nabla}(\boldsymbol{M} \cdot \boldsymbol{H})
$$

Using again the vector identity

$$
\begin{aligned}
\boldsymbol{\nabla}(\boldsymbol{a} \cdot \boldsymbol{b}) & =(\boldsymbol{a} \cdot \boldsymbol{\nabla}) \boldsymbol{b}+(\boldsymbol{b} \cdot \boldsymbol{\nabla}) \boldsymbol{a} \\
& +\boldsymbol{a} \times(\boldsymbol{\nabla} \times \boldsymbol{b})+\boldsymbol{b} \times(\boldsymbol{\nabla} \times \boldsymbol{a})
\end{aligned}
$$

taking $\boldsymbol{a}=\boldsymbol{M}$ and $\boldsymbol{b}=\boldsymbol{H}$, results in

$$
\begin{aligned}
f & =-\frac{1}{2}\{(\boldsymbol{M} \cdot \boldsymbol{\nabla}) \boldsymbol{H}+(\boldsymbol{H} \cdot \boldsymbol{\nabla}) \boldsymbol{M} \\
& +\boldsymbol{M} \times(\boldsymbol{\nabla} \times \boldsymbol{H})+\boldsymbol{H} \times(\boldsymbol{\nabla} \times \boldsymbol{M})\} .
\end{aligned}
$$

However, since there are only uniform static magnetic fields and there are not free currents, we have

$$
\boldsymbol{\nabla} \times \boldsymbol{H}=0,
$$

and

$$
(\boldsymbol{M} \cdot \boldsymbol{\nabla}) \boldsymbol{H}=0 .
$$

We can also see that

$$
\boldsymbol{H} \times(\boldsymbol{\nabla} \times \boldsymbol{M})=0,
$$

since the only component different from zero is $M_{z}(z)$, then $\nabla \times M=0$. Therefore, the force density Eq. (48) reduces to

$$
\boldsymbol{f}_{\text {new } M}=-\frac{1}{2} \mu_{0}(\boldsymbol{H} \cdot \boldsymbol{\nabla}) \boldsymbol{M},
$$

which is not the same as

$$
\boldsymbol{f}^{\prime}=(\boldsymbol{M} \cdot \boldsymbol{\nabla}) \boldsymbol{H},
$$

applicable in the case of a non-uniform $\boldsymbol{H}$ field.

It has been shown elsewhere [23] that the force density Eq. (52) leads to the known result, Eq. (42). This result is a consequence of the usual energy density and vector analysis, and as said before, indicates that the problem of a magnetizable rod inside a solenoid is not equivalent to the problem of a dielectric slab inside a capacitor. The electrostatic force density, Eq. (28), is due to the compression around the electrostatic lines of force, producing a force orthogonal to the interface, while the magnetic force density, Eq. (52), is due to the magnetic tension along the lines of force, and is in the direction of the magnetic field. A magnetic effect truly analogous to the electrostatic case would be a force orthogonal to the lines of magnetic force. We have proposed elsewhere [10] a device that shows such a force. 


\section{Conclusions}

Though the interaction of polarizable and magnetizable matter with electrostatic and magnetostatic fields is the simplest interaction field-matter, it presents conceptual problems that exhibit some inconsistencies. Thus, the usual explanation of the force that pulls a dielectric slab into a parallel-plate capacitor is that it arises from the action of the non-uniform electrostatic field of the fringing field on the electric dipoles of the dielectric. However, the accepted calculation of the force is done with the uniform electrostatic field inside the capacitor. An author [2] has asked how is possible that an electrostatic field can exert a force orthogonal to it.

We offer an alternative explanation based on a momentum balance equation derived from Maxwell's equations. This approach uses Maxwell's stress tensor, which decomposed into principal axes implies a tension along and compression around the lines of force. We have here the original conception of Faraday and Maxwell of the field as a stressed medium. In this way, the possibility of a force orthogonal to the lines of force arises. We argue that it is this type of force acting at the interface that pulls the dielectric slab into the capacitor.

We also argue that the force that pulls a magnetizable bar into a solenoid is due to the tension along the magnetic lines of force. This explains how a uniform magnetic field can exert a net force on magnetic dipoles. Thus, these effects are not analogous, as usually assumed. We have proposed elsewhere [10] a magnetic device in which the force arises from the compression around the magnetic lines of force, being in this way analogous to the electric case.

Thus, our approach, firmly founded on Maxwell's theory and vector calculus, provides interesting insights into the origin of these forces. As an example of these insights, we have that the sucking of water by a laser bean arriving normally to its surface may have a physical explanation in the forces exerted orthogonally to the electric field, since in this case, we have an average electric field parallel to the interface, as in the cases discussed in this work.
1. L. Eyges, The Classical Electromagnetic Field, (AddisonWesley, Reading Mass. 1972) Introduction p.v.

2. S. Margulies, Am. J. Phys. 52 (1984) 515. https://doi. org/10.1119/1.13861

3. C. A. Utreras-Diaz, Am. J. Phys. 56 (1988) 700. https: //doi.org/10.1119/1.15504

4. A. Naini and M. Green, Am. J. Phys. 45 (1997) 877. http: //doi.org/10.1119/1.11075

5. D. J. Griffiths, Introduction to Electrodynamics 3rd edn. (Englewood: Prentice-Hall 1999) pp. 202-205.

6. E. R. Dietz, Am. J. Phys 72 (2004)1499. https://doi. org/10.1119/1.1764563

7. R. Becker, Electromagnetic Field and Interactions (Mineola: Dover 1982) pp. 129-134.

8. J. A. Stratton, Electromagnetic Theory (New York: McGrawHill, 1941) pp. 96-103.

9. W. K. H. Panofsky and M. Phillips, Classical Electricity and Magnetism 2nd edn. (Reading: Addison-Wesley, 1962) pp. 103.

10. J.L. Jiménez-Ramírez, I. Campos-Flores J. A. E. and RoaNeri, Journal of Electromagnetic Analysis and Applications 11 (2019) $2510.4236 /$ jemaa.2019.113003

11. F. Rohrlich, Classical Charged Particles 3rd ed., (World Scientific, Singapore 2007). pp. 54.

12. C. Itzykson, J. Zuber, Quantum Field Theory (McGraw-Hill New York, 1980 (Dover edition, 2005) pp. 7.

13. J. L. Jiménez, I. Campos and M. A. López-Mariño, Eur. Phys. J. Plus, 128 (2013) 46 DOI:10.1140/epjp/i2013-13046-8. 10. 1140/epjp/i2013-13046-8

14. J. L. Jiménez, I. Campos, and M.A. López-Mariño, Eur. Phys. J. Plus, 128 (2013) 129 DOI:10.1140/epjp/i2013-13129-6. 10. 1140/epjp/i2013-13129-6
15. J. C. Maxwell An Elementary Treatise on Electricity 2nd edn (Mineola: Dover 2005). (From 2nd edition of the 1881 book (Oxford: Clarendon Press))

16. J. D. Jackson, Classical Electrodynamics 3rd edn (New York: Wiley and Sons 1999) pp. 212-215.

17. J. L. Jiménez and I. Campos, Advanced Electromagnetism Foundation Theory and Applications ed T W Barret and D Grimes (Singapore: World Scientific 1995)

18. E. J. Konopinski Electromagnetic Fields and Relativistic Particles (New York: McGraw-Hill 1981)

19. I. Campos, J. L. Jiménez and M. A. López-Mariño Electromagnetic momentum balance equation and the force density in material media Revista Brasileira de Ensino de Física 34 (2012) 2303.

20. R. K. Wangsness, Electromagnetic Theory 2nd edn (New York: Wiley and Sons 1986) pp. 165-167, 293-295.

21. J. R. Reitz, F. J. Milford and R. W. Christy, Foundations of Electromagnetic Theory 4th edn (Reading: Addison-Wesley 1993) pp. 115-118, 234-238.

22. I. Campos-Flores, J. L. Jiménez-Ramírez and J.A.E. RoaNeri, Journal of Electromagnetic Analysis and Applications 10 (2018)131.10.4236/jemaa.2018.1010013

23. J. L. Jiménez-Ramírez, I. Campos-Flores and J.A.E. RoaNeri, Journal of Electromagnetic Analysis and Applications 10 (2018) 171. https://doi.org/10.4236/jemaa. 2018.1010013

24. L. D. Landau, E- M. Lifshitz and L. P. Pitaevskii, Electrodynamics of Continuous Media 2nd edn. (Oxford: Pergamon Press 1984) pp. 116-121. 